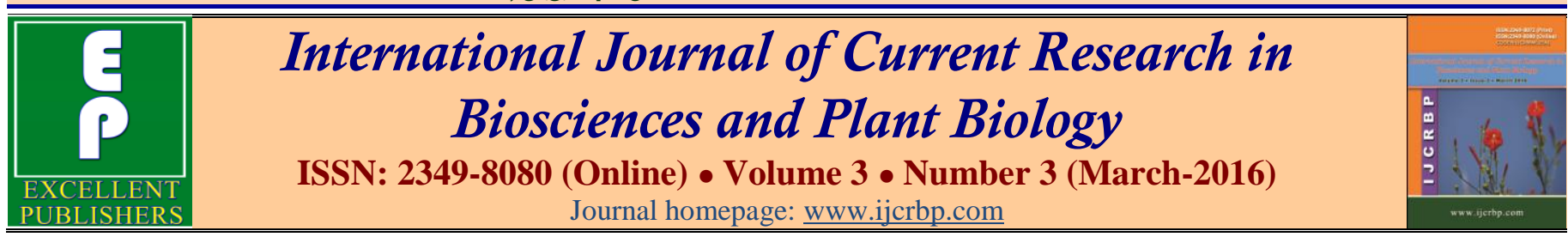

\title{
Impact of Ionizing Radiation of X-Ray Induced Chromosomal Aberration during Spermatogenesis in Aulacophora foveicollis (A Coleopteran)
}

\author{
Sarita Sharma* \\ Department of Zoology, Jawaharlal Nehru College, Bhopal, Madhya Pradesh, India \\ *Corresponding author.
}

\begin{abstract}
Impact of ionizing radiation of X-ray at $1500 \mathrm{r}$ ( roentgen ) has been studied on the spermatogenesis of Aulacophora foveicollis. $\mathrm{X}$-ray induced chromosomal aberrations such as fragmentation, clumping,swelling,stickiness etc. in the spermatogonial, primary spermatocyte and secondary spermatocyte cells in their various stages. The size of cell and nucleus increases and their membrane are generally distorted. The changes in their nucleolar persistence, nucleolar vacuole and perinucleolar ring has also been observed. The effect of X-ray radiation on the further elongating spermatids contain vacuoles which later on fuse together and form an unstained tube like structure. This tube is also lost due to the elongation and condensation of the sperm nucleus. It causes mutagenic effect and create genetic imbalance.
\end{abstract}

\section{Article Info}

Accepted: 29 February 2016

Available Online: 06 March 2016

\section{Keywords}

Chromosomal aberrations

Nucleolar persistence

Perinucleolar ring

Radiation

Spermatids

\section{Introduction}

The cytological studies of spermatogenesis in insects by using X-ray radiation observed the chromosomal aberrations. Many authors such as Murakami and Imai (1974), Haynes et al. (1977), Kumaraswamy and Rajasekarasetty (1979), Carpenter (1991), Sinha and Sinha (1995), Laxmikumari et al. (1996), Verma et al. (1996), Fujiwara et al. (2000), Robinson (2002), Tothova and Marce (2001), Dallai et al. (2002), Chandana et al. (2004), Bakari (2005), Sharma (2008) presents a report on the incidence of visible chromosomal aberrations induced by X-ray radiation.

The aim of the present study is to find out the impact of ionizing radiation of X-ray induced chromosomal aberration during the spermatogenesis in a Coleopteran species, Aulacophora foveicollis.

\section{Materials and methods}

Insect A. foveicollis were collected from agriculture crops and gardens. X-ray exposure of $1500 \mathrm{r}$ was given to the whole insects. The testis was dissected out in the normal saline solution. Its squash and smear preparations were made and stained in the acetocarmine for observation.

\section{Results}

The process of spermatogenesis in A. foveicollis the effect of X-ray radiation of $1500 \mathrm{r}$ was being observed on the cytological features during various stages of the spermatogonial primary spermatocyte and secondary spermatocyte cells.

Spermatogonial cells at the radiation of $1500 \mathrm{r}$

Prophase stage: At the radiation of 1500r the 
nucleus as well as the nuclear membrane increases in size due to the swelling. The chromosomes show little breakage and become thick (Fig. 1).

Metaphase stage: The cell become enlarged and chromosomes become fragmented, condensed and lie in the sticky nucleoplasm (Fig. 2).

Anaphase stage: A few fragments of chromosome fail to reach their respective poles as their movement is obstructed by stickiness of the cytoplasm. However the majority of the chromosomal fragments remain clumped together at their poles (Fig. 3).

Telophase stage: The chromosomes in each daughter cell appear condensed, deeply stained bodies and lie sticking with each other inside the nucleus.

At this stage the nucleolus and the nuclear membrane are reformed (Fig. 4).
1

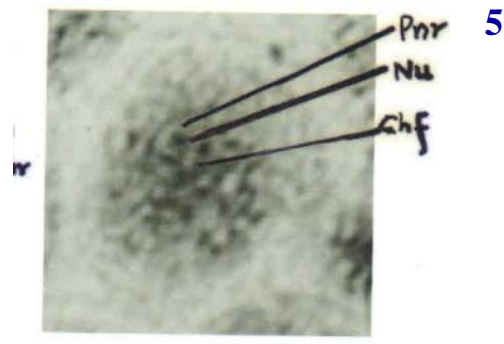

Pro.-1500r

2

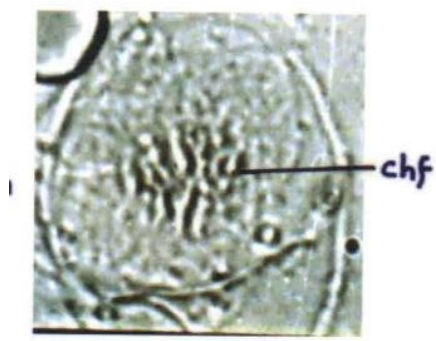

Meta.-1500r

3

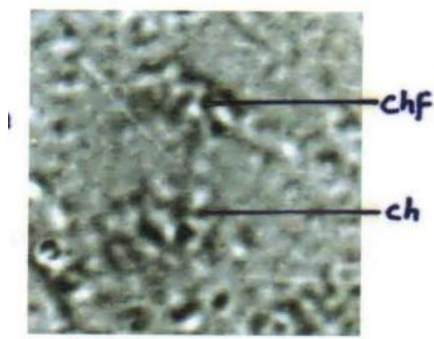

Ana.-1500r

4

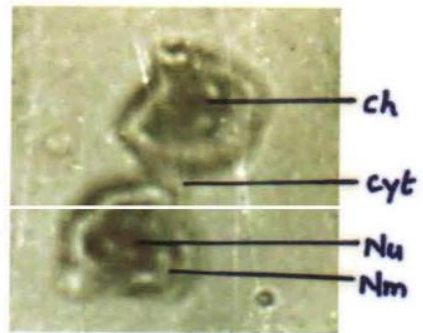

Telo.-1500r

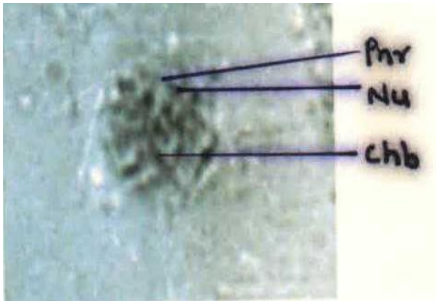

Lept.-1500r

6

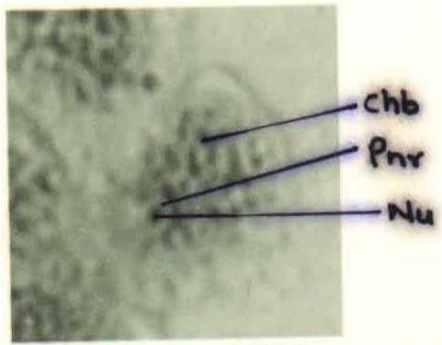

Zygo.- $1500 \mathrm{r}$

7

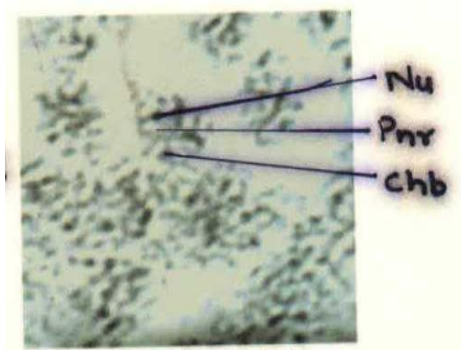

Pach.-1500r

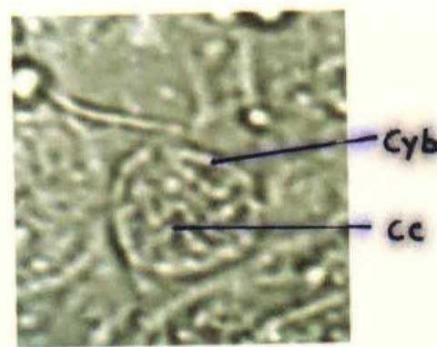

Dipl.-1500r

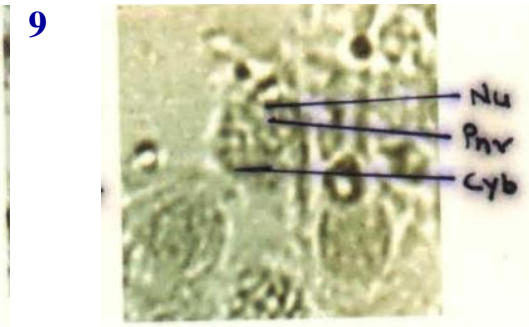

Pro.-1500r

10

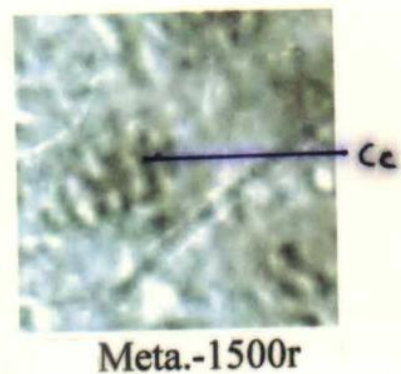

Meta.-1500r

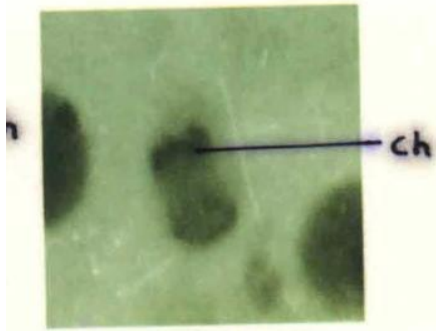

Ana.-1500r

12

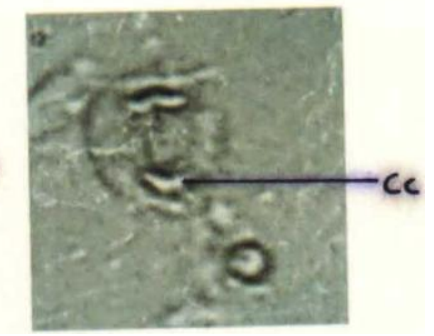

Figs. 1-4: Spermatogonial cells at the radiation of $1500 \mathrm{r}$. Figs. 5-8: primary spermatocyte cells at the radiation of $1500 \mathrm{r}$; Figs. 9-12: secondary spermatocyte cells at the radiation of $1500 \mathrm{r}$. 
13

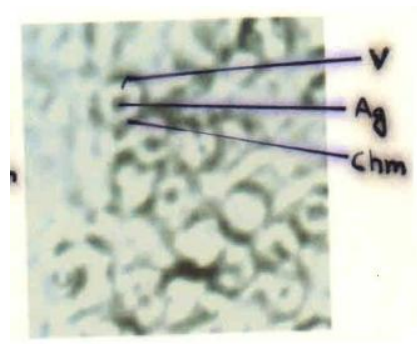

14

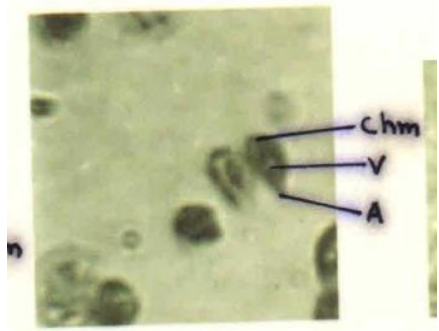

Sp. $-1^{\text {st }}-1500$ r

15

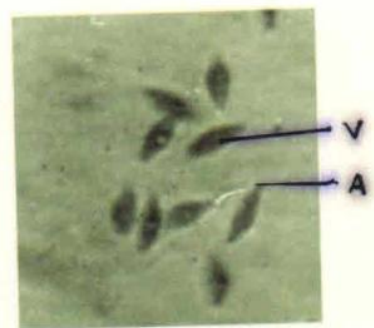

Sp. $-2^{\text {nd }}-1500 \mathrm{r}$

16

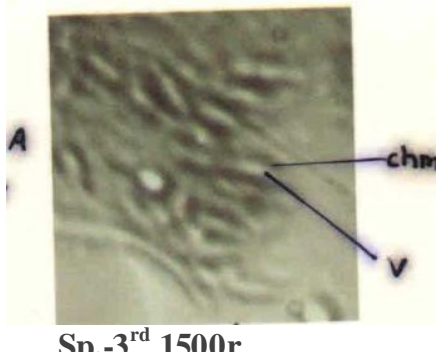

17

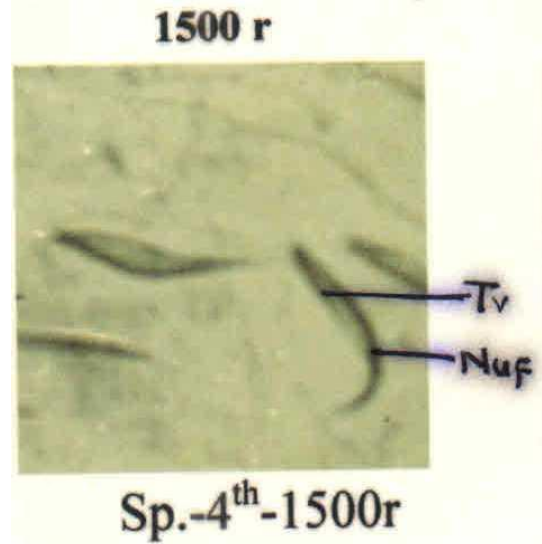

18

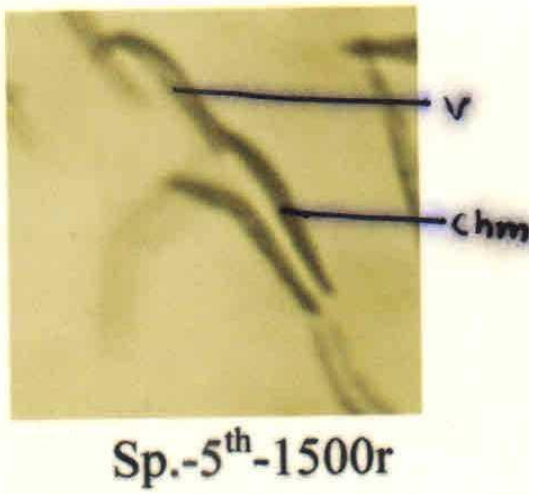

19

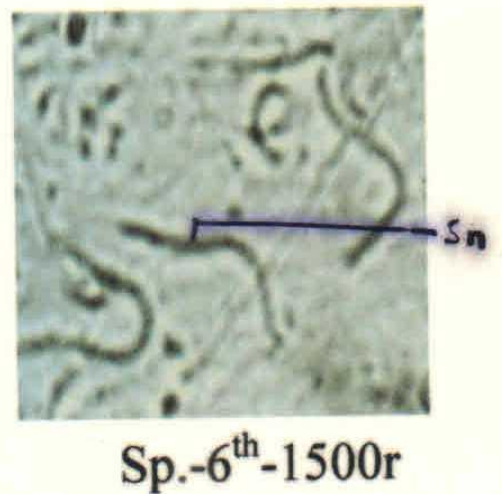

Figs. 13-19: Spermatid, their elongation and sperm at the radiation of $1500 \mathrm{r}$.

\section{Primary spermatocyte cells at the radiation of $1500 \mathrm{r}$}

Leptotene stage: The cell membranes as well as the nuclear membranes are distorted. The cytoplasm become sticky. The nucleus swells up and contains a nucleolus encircled by a perrinucleolar ring. The chromosomal threads show breakage and become thicker (Fig. 5).

Zygotene stage: The chromosomes show breakage and stickiness. The nucleolus is seen surrounded by a perinucleolar ring but the nucleolar vacuole is absent (Fig . 6).
Pachytene stage: The chromosomal loops, break and lie in the sticky cytoplasm. The nucleolus shrinks and is found surrounded by the perinucleolar ring (Fig. 7).

Diplotene stage: The thick cytoplasmic blocks are found arranged in a single row just below the cell membrane. The cytoplasm becomes sticky. The nucleus swells up and its membrane break at certain places. Fragmented and condensed chromosomes lie clumped with each other in the thick cytoplasm (Fig. 8).

Diakinesis stage: The nucleus enlarges and the chromosomes become condensed. The nuclear 
membrane disappears. The chromosomes become condensed. These appear like dot and scattered in the sticky cytoplasm (Fig. 9).

Metaphase stage: The chromosomes condensed and lie dispersed in the thick and sticky cytoplasm (Fig. 10).

Anaphase stage: The chromosomes at both the poles appear clumping with each other (Fig. 11).

Telophase stage: The chromosomes become condensed and lie aggregated at both the poles. This is followed by cytoplasmic division (Fig. 12).

\section{Secondary spermatocyte cells at the radiation of $1500 \mathrm{r}$}

Prophase stage: A row of small cytoplasmic blocks are found arranged below the cell membrane. The nucleus swells up and the chromosomal thread, break in to small pieces. Nucleolus and perinucleolar ring are present (Fig. 13).

Metaphase stage: The chromosomes become much condensed and seen clumped together in the sticky cytoplasm (Fig. 14).

Anaphase stage: Condensed chromosomes moves towards their respective poles (Fig. 15).

Telophase stage: The chromosomes become much condensed and appear as clumped masses at their poles (Fig. 16)

Spermatid, their elongation and sperm at the radiation of $1500 \mathrm{r}$

Spermatid: At this radiation chromatin material becomes condensed with results the size of the nuclear vesicle appears to be increased. The size of the acrosome is enlarged (Fig. 17).

Spermatid $1^{\text {st }}$ elongation: The cell swells up. The elongating spermatid becomes pointed at its anterior and broad at the posterior end. The cell contains well stained chromatin material containing one to two vacuoles within it (Fig. 18)

Spermatid $2^{\text {nd }}$ elongation: One or two small vacuoles are formed with in the uniformly distributed chromatin material of the nucleus (Fig. 19).

Spermatid $3^{\text {rd }}$ elongation: The vacuole of the spermatid inside the cell becomes very much enlarged therefore, the chromatin material is pushed at the margin and aggregated of the cell.

Spermatid $4^{\text {th }}$ elongation: On further elongation the lateral margins of the flattened spermatid nucleus roll-up and fuse together longitudinally like ' trypanosome' flattened structure result on elongated tube like space is enclosed inside it.

Spermatid $5^{\text {th }}$ elongation: The chromatin material becomes granular and lie aggregated in a liner fashion. The elongating single vacuole breaks in three to four vacuoles.

Sperm: A newly formed sperm has a well stained long nucleus having a cytoplasmic tail connected at its posterior end.

\section{Discussion}

Murakami and Imai (1974) observed in Bombyx mori when treated with X-ray (1000r) and the gamma rays also produced chromosomal aberrations. Haynes et al. (1977) in Boll weevil pupa Anthonomus grandis reported that fractional doses of $6250-8000 \mathrm{r}$ of gamma irradiation when administrated to pupa of boll weevil, it sexually sterilized. Rajasekarasetty and Kumaraswamy (1979) in Poekilocerus pictus (Orthoptera) reported that gamma rays induced chromosomal aberrations on short-horned grasshopper when exposed to whole body irradiations of different doses of gamma rays. Carpenter (1991) observed chromosomal aberrations due to radiation in Helicoperva zea (Lepidoptera). Sinha and Sinha (1995) reported that in B. mori the males when irradiated with 500r, 1000r and 2000r formed bivalents chains and ring bivalent in diplotene and diakinesis stages.

Laxmikumari et al. (1996) reported that in B.mori the gamma radiation of $1500 \mathrm{r}$ induced chromosome showing stickiness during spermatogenesis. Verma et al. (1996) observed stickiness of chromosome in meiotic studies during radiation in P.dermondii. Fujiwara his coworkers (2000) in B.mori reported that x-ray irradiations produced chromosomal fragmentation. Tothova and Marce (2001) observed in the male flour moth Ephistia kuchniella (Lepidoptera) induced gamma rays produce chromosomal aberrations at 100Gy. Chandana et al. (2004) reported on radio resistance of Lepidopteran insects at the exposure of $200 \mathrm{~Gy}$ the nuclear size and granulity increases. 
Spermatogonial, primary spermatocyte and the secondary spermatocyte cells of A. foveicollis induce X-ray radiations probably produce some chemical changes in the cytoplasm, nucleoplasm or in the chromosomes is one of the reasons for the stickiness. X-ray irradiation produces changes in the spermatids such as swelling of the cell, the chromosomal disorder etc., which are responsible for producing genetic mutations. Dallai et al. (2002) observed that spermatozoa of A. formicaria show that the sperm nucleus is the characterized by the presence of two lateral grooves which are filled with numerous infoldings of the nuclear envelope. Robinson (2002) used the radiation to specific type of mutations in the sterile insects technology (SIT), Bakari (2005) in a review on IDIDAS (International Database on Insect Disinfestation and Sterilization) about radiation doses for disinfestation and reproductive sterilization in insects and also gave the information on different pest control applications and biological control programmes. Sharma (2008) found that the elongating spermatid nucleus becomes flattened and appear like a 'Leaf' or a 'trypanosome'. Sperms are sensitive to X-ray and their fertility is depressed cause several times more genetic mutations.

\section{Conclusion}

Ionizing radiation of $\mathrm{X}$-ray induced chromosomal abnormalities like chromosomal aberrations during spermatogenesis and cause mutagenic effect and create genetic imbalance. There is every possibility that these effects are passed onto future generations also.

\section{Conflict of interest statement}

Authors declare that they have no conflict of interest.

\section{Acknowledgement}

Author is thankful to the Head of the Department of Zoology, Govt. V.Y.T.P.G. College, Durg (CG) for providing necessary facilities.

\section{References}

Bakari, A., Heather, N., Hendrichs, J., Ferris, I. 2005. Fifty years of radiation biology in entomology: Lessons learned from IDIDAS. Ann. Entomol. Soc. Amer. 98(1), 1-12.

Chandana, S., Dwarkanath, B.S., Seth,R.K., Khaitan, D., Adhikari, J.S., Jain,V., 2004. Radiation responses of SF9, a highly radio resistant Lepidopteran insect cell line. Int. J. Rad. Biol. 80(4), 301-315.

Carpenter, J.E., 1991. Effect of radiation dose on the incidence of visible chromosomal aberrations in Helicoperva zea (Lepidoptera: Noctuidae). Environ. Entomol. 20, 14571459.

Dallai, R., Lupetti, P., Carapelli, A., Frati, F., Afzelius, B., 2002. Sperm structure and spermiogenisis in Atelura formicaria Hayden (Zygentoma, Insecta). Acta Zool. 83(3), 245.

Fujiwara, H., Nakazato, Y., Okazaki, S., Ninaki, O., 2000. Stability and telomere structure of chromosomal fragments in two different mosaic strains of silkworm, Bombyx mori. Zool. Sci. 17(6), 743-750.

Haynes, J.W., Normammitlin, T.B., Davich, J.R., Dawson, W.L., Mcgovern, Mckibben, G.H., 1977. Sterilization of boll weevil pupae with fractionated doses of gamma irradiation. 21, 57-62.

Kumarsawamy, K.R., Rajasekarasetty, M.R., 1979. Gamma rays induced chromosomal aberrations in Poekilocerus picta (Orthoptera). Indian J. Hered. 8, 11.

Laxmikumari, B., Jayaprakash., Ananthanarayan, S.R.,1996. Effect of gamma radiation on meiotic chromosomes of Bombyx mori L. J. Cytol. Genet. 31 (1), 27-30.

Murakami, A., Imai, H.J., 1974. Cyological evidence for holocentric chromosomes of the silkworms, Bombyx mori and B. mandarina (Bombycidae- Lepidoptera) J. Chromosoma. 47(2), 167-178.

Robinson, A.S., 2002. Mutations and their uses in insect control. Mutant Res. 511(2), 113-32.

Sharma, S., 2008. Studies on behaviour of chromosomes during spermatogenesis in A. foveicollis. NJLS.3(3),22-25.

Sinha, R.K., Sinha, S.P., 1995. Effect of mutagens on chiasma frequency in Bombyx mori L. (Lepidoptera: Bombycidae) Cytol. Genet. 30(1), 91-94.

Tothova, A., Marec, F., 2001. Chromosomal Principle of radiation-induced F1 sterility in Ephestia kuchniella (Lepidoptera: Pyralidae). Genome. 44(2), 172-184

Verma, R.C., Reddy, B.M., and Shevade,A.1996. Meiotic studies in a radiation induced mutant in Phlox dermondii. J. Cytol. Gene. 31(1), 23 -25.

\section{How to cite this article:}

Sharma, S., 2016. Impact of ionizing radiation of X-ray induced chromosomal aberration during spermatogenesis in Aulacophora foveicollis (A Coleopteran). Int. J. Curr. Res. Biosci. Plant Biol. 3(3), 48-52. doi: http://dx.doi.org/10.20546/ijcrbp.2016.303.009 